\title{
An NMR-based metabonomic investigation on effects of milk and meat protein diets given to 8-year-old boys
}

\author{
Hanne Christine Bertram ${ }^{1}$, Camilla Hoppe ${ }^{2}$, Bent O. Petersen ${ }^{3}$, Jens $\varnothing$. Duus $^{3}$, Christian Mølgaard $^{2}$ and \\ Kim F. Michaelsen ${ }^{2}$ \\ ${ }^{1}$ Danish Institute of Agricultural Sciences, Department of Food Science, Research Center Foulum, P.O. Box 50, DK-8830 Tjele, \\ Denmark \\ ${ }^{2}$ Department of Human Nutrition, University of Copenhagen, Rolighedsvej 30, DK-1958 Frederiksberg C, Denmark \\ ${ }^{3}$ Carlsberg Laboratory, Gamle Carlsberg Vej 10, DK-2500 Valby, Denmark
}

(Received 11 October 2006 - Revised 15 November 2006 - Accepted 16 November 2006)

\begin{abstract}
The objective of the study was to investigate the ability of an NMR-based metabonomic approach, applied to biofluids, to explore and identify overall exogenous and endogenous biochemical effects of a short-time high intake of milk protein or meat protein given to prepubertal children, the aim being to compare relative differences and not an absolute quantification. A total of twenty-four 8-year-old boys were asked to take $53 \mathrm{~g}$ protein as milk $(n 12)$ or meat daily $(n 12)$. At baseline and after $7 \mathrm{~d}$, urine and serum samples were collected and high-resolution ${ }^{1} \mathrm{H}$ NMR spectra were acquired on these using a $800 \mathrm{MHz}$ spectrometer. The milk diet reduced the urinary excretion of hippurate, while the meat diet increased the urinary excretion of creatine, histidine and urea. The NMR measurements on serum revealed minor changes in the lipid profile, which most probably should be ascribed to an increase in the content of SCFA in the blood after consumption of the milk diet. The meat diet had no effect on the metabolic profile of serum. The study for the first time demonstrates the capability of proton NMR-based metabonomics to identify the overall biochemical effects of consumption of different animal proteins. The urine metabolite profile is more susceptible to perturbations as a result of short diet interventions than the serum metabolite profile. The milk diet-induced reduction in urinary excretion of hippurate suggests alterations in gut microflora, which may be useful information for further studies elucidating the effects of bioactive components in milk.
\end{abstract}

Urine: Serum: Proton NMR spectroscopy: Animal protein: Children: Metabolomics: Hippurate: Short-chain fatty acids

Proteins are an essential component of the human diet. When expressed per $\mathrm{kg}$ body weight, the protein intake in young children is very high, typically two to three times higher than in adults and also two to three times higher than the physiological requirement (Rolland-Cachera et al. 1999). It is unknown whether this has positive or negative long-term effects. It has been outlined that a diet rich in animal protein lacks alkalizing effects and therefore may have a negative effect on $\mathrm{Ca}$ balance and bone mineral density (Demigné et al. 2004) in some studies, but dietary protein is also positively associated with bone mineral density in children (Hoppe et al. 2000) and adults (Bonjour et al. 1997; Teegarden et al. 1998). However, in general the metabolic effects of a high-protein diet are poorly understood, especially in children. In addition, the knowledge of the significance of protein source is also very limited. It was demonstrated that a milk protein diet increases serum insulin-like growth factors (Hoppe et al. 2004) as well as insulin and insulin resistance in prepubertal boys (Hoppe et al. 2005). However, it has not been studied any further to what extent a highprotein diet and protein source may also have other biochemical effects.
Proton NMR provides concurrent detection of all hydrogencontaining molecules in a sample without pretreatment, and it is widely recognized that NMR-based metabonomics is an excellent tool in pharmaceutical studies for detection of biochemical effects without any a priori knowledge (Lindon et al. 2006). Within the field of nutrition the use of NMRbased metabonomics is more rare, however, the technique has exciting opportunities for elucidating how diet elicits changes in metabolite profile (Gibney et al. 2005), and applications of NMR-based metabonomic for elucidating the biochemical effects of dietary bioactive compounds in both animals (Solanky et al. 2003a; Bertram et al. 2006) and man (Solanky et al. 2003b; Daykin et al. 2005; Wang et al. 2005) have been demonstrated. Thus, NMR-based metabonomics could be an effective tool for investigating possible biochemical effects associated with protein source and a high-protein diet, as the technique enables a screening without any a priori knowledge of expected outcome.

The aim of the present study was to investigate the ability of NMR-based metabonomics to identify exogenous and endogenous biochemical effects of a milk protein and a meat protein diet given to prepubertal children in an explorative

\footnotetext{
Abbreviations: PLS-DA, partial least square regression discriminant analysis; TMAO, trimethylamine- $N$-oxide; TOCSY, $2 \mathrm{D}{ }^{1} \mathrm{H}-{ }^{1} \mathrm{H}$ total correlation. * Corresponding author: Dr Hanne C. Bertram, fax +45 899915 64, email HanneC.Bertram@agrsci.dk
} 
way. We therefore present a proton NMR-based metabonomics approach applied on urine and serum samples from an intervention study, in which 8-year-old boys were given a high amount of animal protein as either milk or meat for 1 week (Hoppe et al. 2004).

\section{Materials and methods}

\section{Subjects}

As previously described (Hoppe et al. 2004), twenty-four 8 -year-old boys were asked to take about $53 \mathrm{~g}$ protein daily, twelve boys as 1.51 skimmed milk, and twelve other boys as $250 \mathrm{~g}$ low-fat meat. The milk and meat supplements were provided to the children from the Department of Human Nutrition. In addition, they were asked to eat ad libitum their normal diet.

The subjects were recruited in the following manner: boys born between October and December 1992 were drawn at random from the Central Personal Register, and were invited to participate in the study. Children with a habitual milk intake of $500 \mathrm{ml} / \mathrm{d}$ or less, who were willing to increase their intake of milk or meat considerably during a week, were eligible for the study. Children with chronic illnesses, and children who suffered from any condition likely to affect their protein metabolism or growth were excluded from the study. From a total of 313 invited subjects, thirty agreed to participate, and twentyeight were eligible for the study. Of the twenty-five boys who were the first to agree to participate in the study, fourteen were eligible and twelve completed the intervention with milk, and of the remaining fifteen boys, fourteen were eligible and twelve completed the intervention with meat. All participants were Caucasian. Subjects and their parents received oral and written information about the study, and the parents gave their written consent. The participants (with their parents) kept a $3 \mathrm{~d}$ weighed food record prior to the intervention day (day -3 to day -1 ) and during the last $3 \mathrm{~d}$ of the intervention (day 4 to day 7). Blood serum samples were drawn from venepuncture after an overnight fast at baseline and after $7 \mathrm{~d}$ of intervention. The serum was kept frozen at $-80^{\circ} \mathrm{C}$ for 6 years until analysis in 2006. Urine was collected for $24 \mathrm{~h}$ at baseline and after $7 \mathrm{~d}$ of intervention. Urine was collected in containers with $10 \mathrm{ml} 1 \mathrm{M}-\mathrm{HCl}$ added, and urine samples were subsequently kept frozen at $-20^{\circ} \mathrm{C}$ for 6 years until analysis in 2006 .

\section{NMR measurements}

Prior to the measurements the serum samples were thawed and $200 \mu \mathrm{l}$ aliquots were mixed with $400 \mu \mathrm{l} \mathrm{D}_{2} \mathrm{O}$. Urine samples were thawed, $\mathrm{pH}$ of the urine was adjusted to 4 by adding the appropriate amount of concentrated $\mathrm{HCl}$ or $\mathrm{NaOH}$, and $400 \mu \mathrm{l}$ aliquots were mixed with $200 \mu \mathrm{l} \mathrm{D}_{2} \mathrm{O}$. The NMR measurements were performed at $298 \mathrm{~K}$ on a Varian Inova 800 spectrometer (Varian, Palo Alto, CA, USA) equipped with a $5 \mathrm{~mm}{ }^{1} \mathrm{H}$ observe probe. ${ }^{1} \mathrm{H}$ NMR spectra were obtained using a standard $1 \mathrm{D}$ pulse sequence (relaxation delay $-90^{\circ}-\mathrm{t}_{1}-90^{\circ}-\mathrm{t}_{\mathrm{m}}-90^{\circ}$-acquire free induction decay). Water suppression was applied using the SWET scheme, which suppresses the water magnetization by spatial scrambling (Wu \& Otting, 2005). A total of 128 transients were collected into 32000 data points using a pulse recycle time of $2 \mathrm{~s}$. A spectral width of $13000 \mathrm{~Hz}$ was used. All spectra were referenced to the methyl group of creatine at $3.04 \mathrm{ppm}$. The spectra were subdivided into $0.005 \mathrm{ppm}$ integral regions and integrated, reducing each spectrum into 1840 independent variables in the region $0 \cdot 18-4 \cdot 4$ and $5 \cdot 1-10 \cdot 0 \mathrm{ppm}$. The reduced spectra in the region $0 \cdot 18-4 \cdot 4$ and $5 \cdot 1-10 \cdot 0$ ppm were normalized to the whole spectrum to remove any concentration effects, and further analysis was performed using the Unscrambler software version 9.2 (Camo, Oslo, Norway). Principal component analysis was applied to the centred data to explore any clustering behaviour of the samples, and partial least square regression discriminant analysis (PLS-DA) was performed to explore intrinsic biochemical dissimilarities between baseline and after $7 \mathrm{~d}$ of intervention. During all regressions, Martens uncertainty test (Martens \& Dardenne, 1998) was used to eliminate noisy variables, and all models were validated using full cross-validation (Martens \& Martens, 2000).

To aid spectral assignment 2D ${ }^{1} \mathrm{H}-{ }^{1} \mathrm{H}$ total correlation (TOCSY), 2D ${ }^{1} \mathrm{H}-{ }^{1} \mathrm{H}$ NOESY and 2D ${ }^{1} \mathrm{H}-{ }^{13} \mathrm{C}$ HSCQ spectra were recorded on selected urine samples using water suppression. The TOCSY spectra were acquired with a spectral width of $8000 \mathrm{~Hz}$ in both dimensions, 4000 data points, 512 increments with thirty-two transients per increment and $80 \mathrm{~ms}$ spinlock. The NOESY spectra were acquired with a similar size and number of data points as the TOCSY and a mixing time of $600 \mathrm{~ms}$. The HSCQ spectra were acquired with a spectral width of $8000 \mathrm{~Hz}$ in the F2 dimension and $28149 \mathrm{~Hz}$ in the F1 dimension, a data matrix with a size of $2400 \times 512$ data points and sixty-four transients per increment.

\section{Results}

Urine

Figure 1 shows a typical ${ }^{1} \mathrm{H}$ NMR spectrum acquired on a urine sample. Several resonances are observed, which were assigned combining the information provided by the $2 \mathrm{D}$ experiments with literature values (Lehnert \& Hunkler, 1986; Fan, 1996; Lindon et al. 1999). In order to investigate possible metabolic differences between sample classes, a principal component analysis was performed on the NMR spectra. A scatter plot of score 1 versus score 2 for mean-centred data (Fig. 2) shows a clear clustering into samples taken before and after the $7 \mathrm{~d}$ of meat and milk diet interventions, respectively.

PLS-DA, which is more focused on discriminating between pre-defined classes than principal component analysis, was performed on the ${ }^{1} \mathrm{H}$ NMR spectra to identify the metabolic differences between baseline and after $7 \mathrm{~d}$ intervention. The first two components in the PLS-DA could explain 67 and $71 \%$ of the variance in Y, for the milk and meat diets, respectively, and for both milk and meat diets the PLS-DA score plots of the first and second component show a clear separation of baseline samples and samples taken after intervention along the first component (Fig. 3(a, c)). The regions of the NMR spectrum that most strongly influence separation between baseline and after diet intervention are evident in the first X-loading (Fig. 3(b, d)). The discrimination between baseline samples and samples taken after milk intervention can mainly be ascribed to a decrease in the spectral regions at 4.03, 7.56, 7.65 and 7.84 ppm (Fig. 3(b)), which has been identified as hippurate. For the meat diet, the main differences observed before and after intervention is in the spectral 


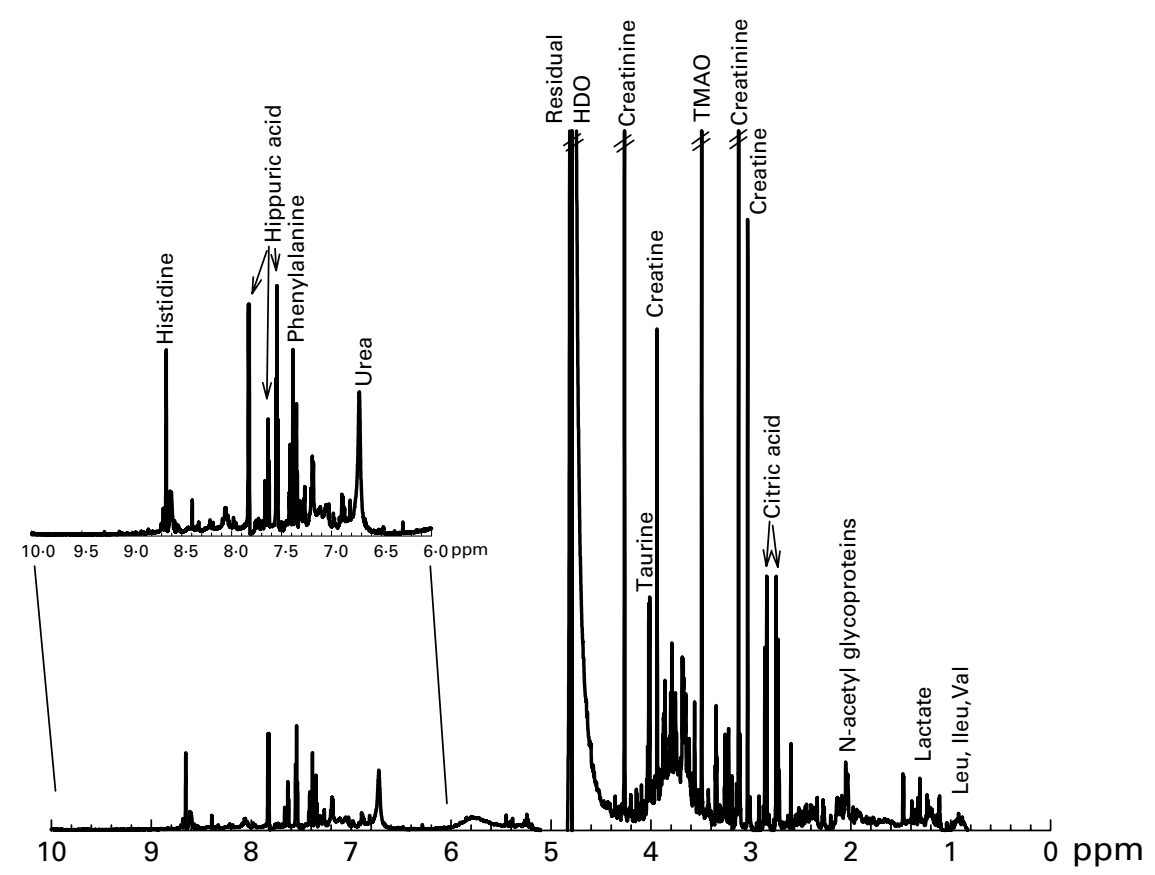

Fig. 1. $800 \mathrm{MHz}{ }^{1} \mathrm{H}$ NMR spectra of a representative baseline urine sample. For details of procedures, see p. 759. HDO, residual water signal; lleu, isoleucine; Leu, leucine; TMAO, trimethylamine- $N$-oxide; Val, valine.

regions at $3 \cdot 13,3 \cdot 52$ and $4.28 \mathrm{ppm}$, which decrease in intensity upon intervention, and the spectral regions at 3.04, 3.88, 3.96, $6.74,7.43$ and $8.69 \mathrm{ppm}$, which increase in intensity upon intervention (Fig. 3(d)). The resonance at $3.52 \mathrm{ppm}$ has been identified as trimethylamine- $N$-oxide (TMAO), and the baseline sample positioned in the upper left corner of the scoreplot (Fig. 3(c)) is characterized by a high intensity of this signal. The spectral regions at 3.13 and $4.28 \mathrm{ppm}$, which decrease slightly upon meat intervention, have been identified as creatinine, while the spectral regions at 3.04 and $3.96 \mathrm{ppm}$, which increase upon meat intervention, have been identified as creatine. The positive value of the resonance at $6.74 \mathrm{ppm}$ reveals an increase in urea in the urine upon the meat intervention, while the positive values of the resonances at 7.43 and 8.69 can be ascribed to an increase in histidine in the urine

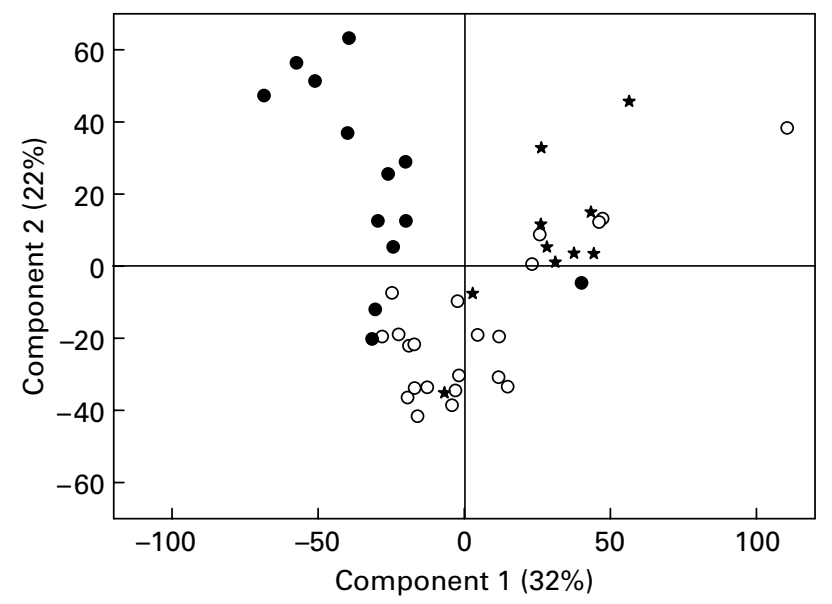

Fig. 2. Principal component analysis score plot showing the two first principal components for urine samples taken before intervention $(\bigcirc)$, and after the meat $(\bullet)$ and milk (*) diet interventions. For details of procedures, see p. 759. upon the meat intervention. It has not been possible to identify the increase in the $3.88 \mathrm{ppm}$ signal, which originates from $\mathrm{CH}$ or $\mathrm{CH}_{3}$ protons.

\section{Blood serum}

A representative ${ }^{1} \mathrm{H}$ NMR spectrum acquired on a serum sample is shown in Fig. 4. In order to investigate possible metabolic differences between sample classes, a principal component analysis was performed on the serum spectra. While a scatter plot of score $1 v$. score 2 does not show any evident clustering (data not shown), a scatter plot of score 2 $v$. score 3 for mean-centred data shows no separation of samples taken before intervention and after meat intervention but a tendency for a separation of samples taken after the milk intervention (Fig. 5). PLS-DA was performed on the ${ }^{1} \mathrm{H}$ NMR spectra acquired on serum to identify the metabolic differences between baseline and after $7 \mathrm{~d}$ intervention. For the meat diet, PLS-DA could not discriminate between baseline samples and samples taken after intervention (Fig. 6(a)) and the first two components could only explain $26 \%$ of the variance in Y. For the milk diet the first two components could explain $40 \%$ of the variance in $\mathrm{Y}$, and a better discrimination is obtained (Fig. 6(b)). The first X-loading reveals that the discrimination between baseline samples and samples taken after $7 \mathrm{~d}$ of the milk diet can be ascribed to increases in the intensity of spectral regions that can be assigned to lipid protons (Fig. 6(c)).

\section{Discussion}

NMR-based metabonomics enables a screening for perturbations in molecular concentrations in biofluids without any $a$ priori knowledge, and thereby without any hypothesis-induced 

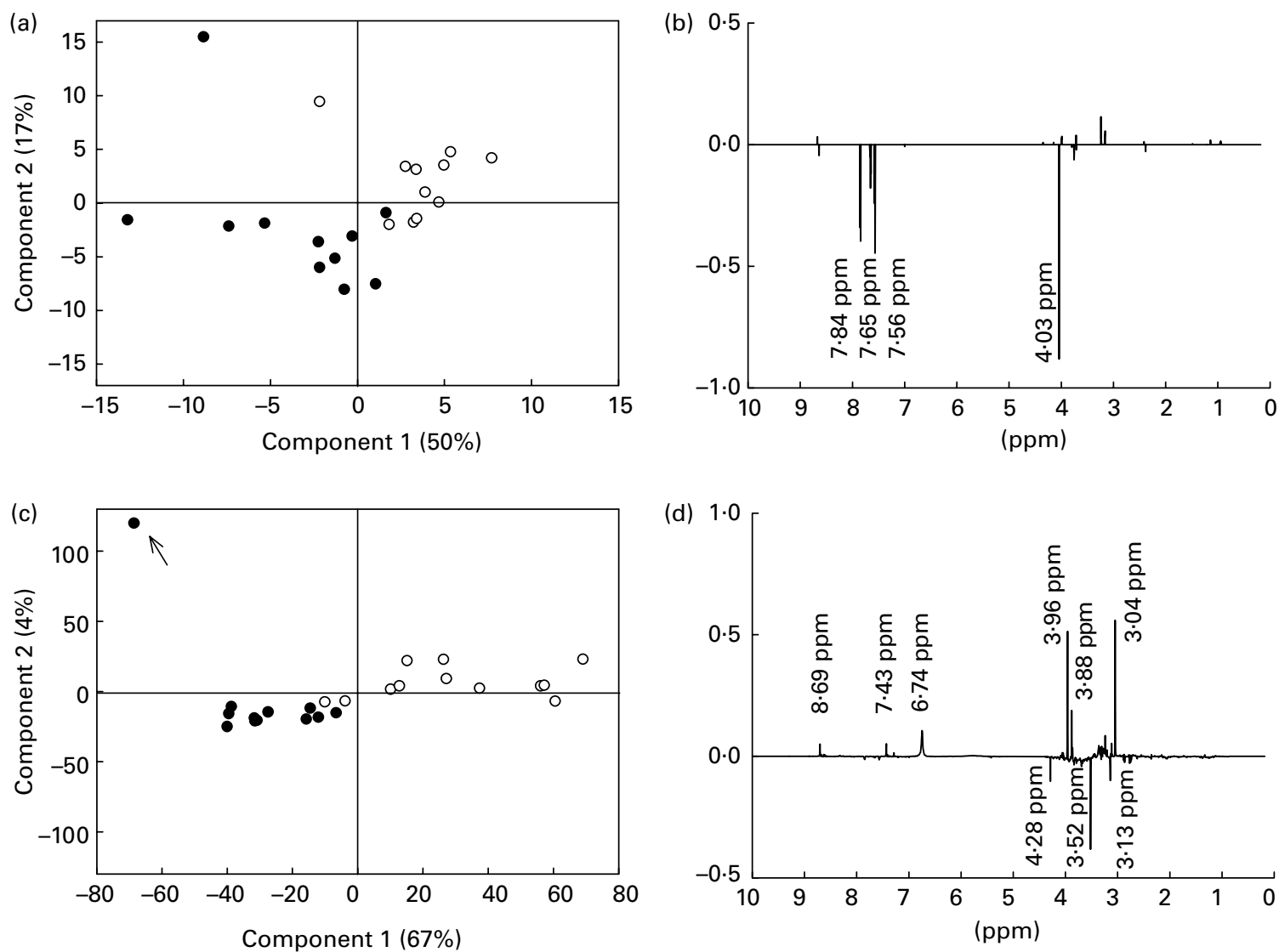

Fig. 3. (a), Partial least square regression discriminant analysis (PLS-DA) score plot from analysis of NMR spectra obtained on baseline urine samples ( $\bullet$ ) and after milk intervention (O). (b), The first X-loading of the PLS-DA shown in (a). (c), PLS-DA score plot from analysis of NMR spectra obtained on baseline urine samples $(\bullet)$ and after meat intervention $(O)(\lambda$, outlier described on p. 760, which is characterized by a high intensity of the trimethylamine- $N$-oxide signal at $3.52 \mathrm{ppm}$ ). (d), The first X-loading of the PLS-DA shown in (c). For details of procedures, see p. 759.

restrictions. While several NMR-based metabonomic studies have been reported for toxicological or pharmaceutical applications (Lindon et al. 2006), applications within the field of nutrition are more rare. Although other dietary changes might contribute to the findings, the present study for the first time

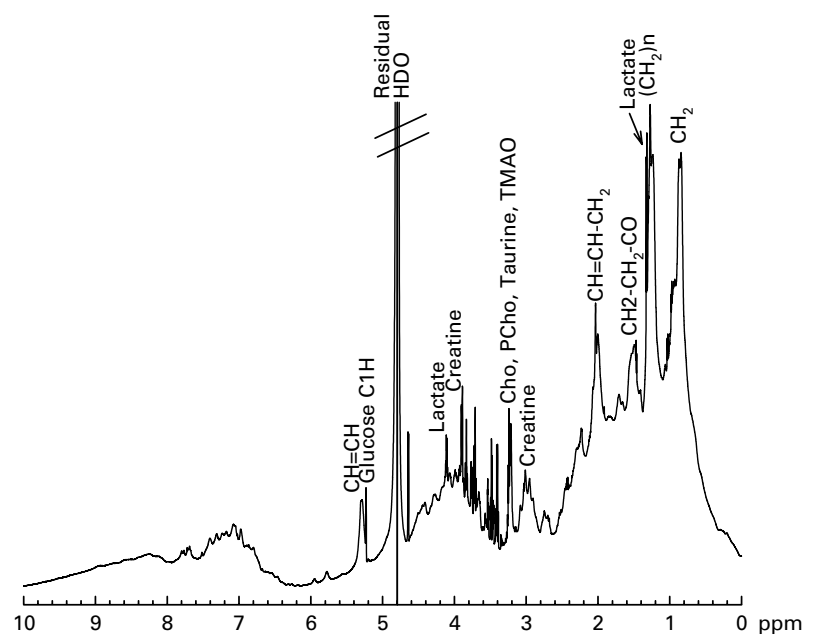

Fig. 4. $800 \mathrm{MHz}{ }^{1} \mathrm{H}$ NMR spectra of a representative baseline blood serum sample. For details of procedures, see p. 759 . Cho, choline; HDO, residual water signal; PCho, phosphocholine; TMAO, trimethylamine- $\mathrm{N}$-oxide. investigated the ability of NMR-based metabonomics to identify exogenous and endogenous biochemical effects of a milk protein and a meat protein diet given to prepubertal children by measurements on blood serum and urine samples from an intervention study, in which 8-year-old boys were given animal

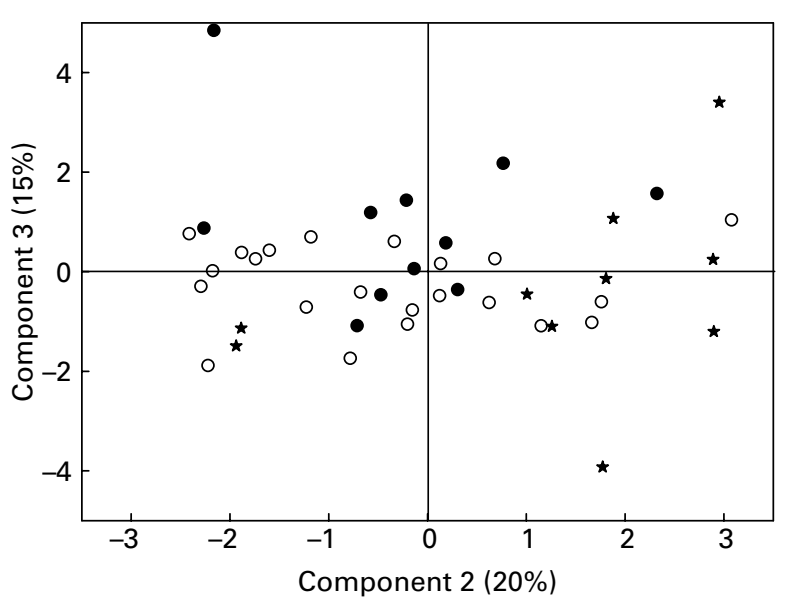

Fig. 5. Principal component analysis score plot showing the two first principal components for serum samples taken before intervention (O), and after the meat $(\bullet)$ and milk $(*)$ diet interventions. For details of procedures, see p. 759 . 

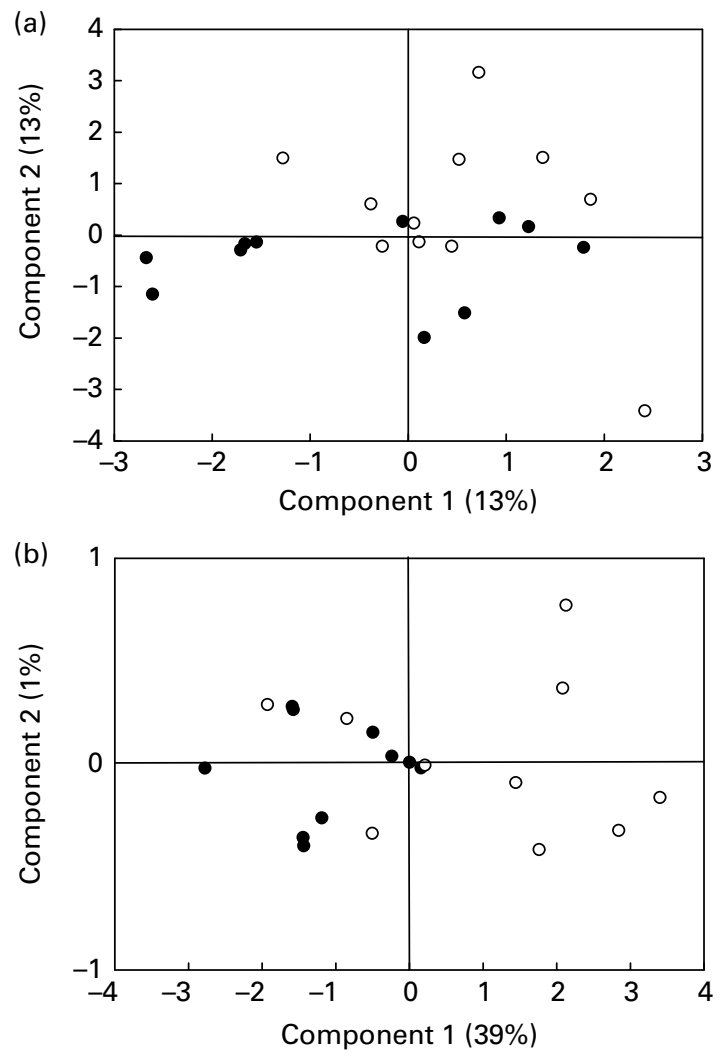

(c)

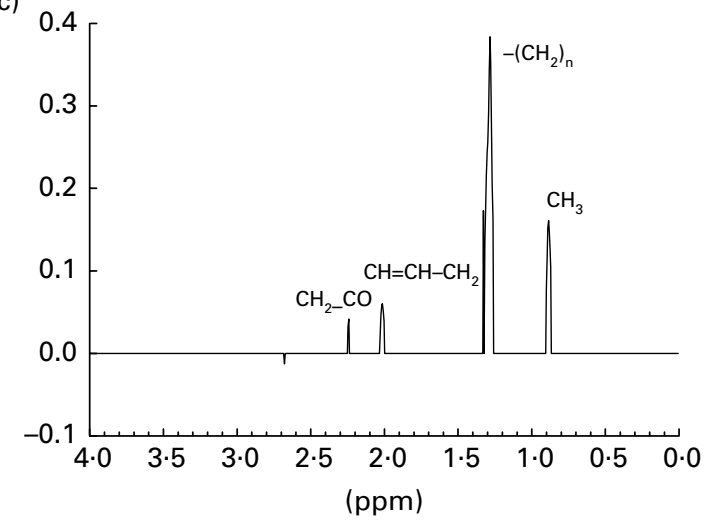

Fig. 6. (a), Partial least square regression discriminant analysis (PLS-DA) score plot from analysis of NMR spectra obtained on baseline serum samples $(\bullet)$ and after milk intervention $(\mathrm{O})$. (b), PLS-DA score plot from analysis of NMR spectra obtained on baseline urine samples $(\bullet)$ and after meat intervention (O). (c) The first X-loading of the PLS-DA shown in (b). For details of procedures, see p. 759.

protein as either milk or meat for 1 week. The aim of the study was not absolute quantification but to compare relative differences, and pronounced effects of the two diets were observed on the urine profile. The milk diet reduced the urinary excretion of hippurate. Hippurate is mainly derived via gut microfloral breakdown of plant phenolics and aromatic amino acids (Phipps et al. 1998; Williams et al. 2002; Mulder et al. 2005). Accordingly, the present findings suggest changes in the gut microfloral metabolism upon an intensive milk diet. The origin of this change remains unknown, as the milk diet was not associated with a major change in dietary intake of aromatic amino acids. Nevertheless, studies on rats have shown a complex relationship between diet and urinary hippurate excretion (Phipps et al. 1998), and the interaction between available substrates and the metabolic capability of the intestinal microflora to produce hippurate is yet not understood. Further studies elucidating the implications of urinary hippurate excretion would be of great interest.

The intervention with the meat diet increased the urinary excretion of creatine, which is in agreement with the fact that meat is the primary dietary creatine source (Barr \& Rideout, 2004). Lately many studies have been conducted with the aim of evaluating the possibility that creatine could function as an ergogenic aid (Lemon, 2002), and it has been observed that adopting a vegetarian diet reduced the muscle creatine concentration (Lukaszuk et al. 2002). However, there has been little focus on the significance of creatine in children's diet. Urinary content of histidine also increased upon the intervention with the meat diet, which is consistent with the fact that meat is the primary histidine source (Hipkiss, 2005). It has been suggested that the dipeptide carnosine, which contains histidine, possesses beneficial effects such as an anti-glycating effect, a regulatory effect on blood glucose level and protection against oxidative damage (Hipkiss, 2005).

TMAO also appeared in the loadings discriminating between baseline urine samples and samples taken after meat intervention. However, this can be ascribed to the fact that a single subject contained a high amount of TMAO in his urine baseline sample (subject indicated with an arrow in Fig. 2(c)). TMAO is often seen after a high intake of fish (Lenz et al. 2004), and the food record prior to the intervention verified a high fish intake at the time of the baseline sampling which explains the elevated concentration of TMAO in the urine from this boy.

In contrast to urine samples, minor differences were observed in the blood serum metabolite profile, and for the meat diet no effects of the intervention could be spotted. This should probably be ascribed to the fact that blood is narrowly controlled in terms of composition and less susceptible to perturbations due to short interventions and that the samples were taken in the morning after at least $10 \mathrm{~h}$ fasting. The spectra on serum were dominated by signals from lipoproteins and lipids, and for the milk diet increases in signal intensities of lipid signals $\left(\mathrm{CH}_{3},\left(\mathrm{CH}_{2}\right)_{n}, \mathrm{CH}=\mathrm{CH}-\mathrm{CH}_{2}\right.$ and $\left.\mathrm{CH}_{2}-\mathrm{CO}\right)$ were observed upon the intervention when compared with baseline. The effect on $\mathrm{CH}_{2}-\mathrm{CO}$ suggests a higher number of fatty acids in serum upon the milk diet, which probably could be ascribed to the presence of additional SCFA in which milk is known to be particularly rich (Garnsworthy et al. 2006). Further studies compiling a complete fatty acid profile of serum in relation to milk consumption could be of great interest for a further understanding of the role of milk intake on serum lipid profile.

It has been claimed that diets low in carbohydrate and rich in protein and lipid are effective in weight loss, and numerous books on the topic have followed, the most well known being Dr. Atkins' New Diet Revolution (Atkins, 1998). According to Atkins' book, weight is lost on the ad libitum low-carbohydrate diet because of increased energy expenditure. However, this has yet not been proven, and mechanisms that could explain weight loss as a result of a diet low in carbohydrate still need to be proven (Astrup et al. 2004). In the present study we did no observations that could attribute 
directly to understanding a possible weight loss as a result of increasing the protein proportion of the diet.

In conclusion, the present study for the first time demonstrated how information on the overall biochemical effects of consumption of different animal proteins can be identified using proton NMR-based metabonomics. The milk dietinduced reduction in urinary excretion of hippurate demonstrates alterations in gut microflora, and further studies are needed to elucidate the significance of the present finding.

\section{Acknowledgements}

The Danish Technology and Production Research Council (FTP) is thanked for financial support through the project 'NMR-based Metabonomics on Tissues and Biofluids' (project no. 274-05-339). The $800 \mathrm{MHz}$ spectra were obtained using the Varian Unity Inova spectrometer of the Danish Instrument Center for NMR Spectroscopy of Biological Macromolecules. Professor Rasmus Bro is greatly acknowledged for his guidance on the multivariate data analysis.

\section{References}

Astrup A, Larsen TM \& Harper A (2004) Atkins and other low-carbohydrate diets: hoax or an effective tool for weight loss? Lancet 364, 897-899.

Atkins RC (1998) Dr. Atkins' New Diet Revolution. New York: Simon \& Schuster.

Barr SI \& Rideout CA (2004) Nutritional considerations for vegetarian athletes. Nutrition 20, 696-703.

Bertram HC, Bach Knudsen KE, Serena A, Malmendal A, Nielsen NC, Fretté XC \& Andersen HJ (2006) NMR-based metabonomic studies reveal changes in the biochemical profile of plasma and urine from pigs fed high-fibre rye bread. Br $J$ Nutr 95, 955-962.

Bonjour JP, Schurch MA \& Rizzoli R (1997) Proteins and bone health. Pathol Biol (Paris) 45, 57-59.

Daykin CA, Van Duynhoven JPM, Groenewegen A, Dachtler M, Van Amelsvoort JMM \& Mulder TPJ (2005) Nuclear magnetic resonance spectroscopic based studies of the metabolism of black tea polyphenols in humans. J Agric Food Chem 53, $1428-1434$.

Demigné C, Sabboh H, Puel C, Rémésy C \& Coxam V (2004) Organic anions and potassium salts in nutrition and metanolism. Nutr Res Rev 17, 249-258.

Fan TW-M (1996) Metabolite profiling by one- and two-dimensional NMR analysis of complex mixtures. Progr Nucl Magn Reson 28, $161-219$.

Garnsworthy PC, Masson LL, Lock AL \& Mottram TT (2006) Variation of milk citrate with stage of lactation and de novo fatty acid synthesis in dairy cows. J Dairy Sci 89, 1604-1612.

Gibney MJ, Walsh M, Brennan L, Roche HM, German B \& van Ommen B (2005) Metabolomics in human nutrition: opportunities and challenges. Am J Clin Nutr 82, 497-503.

Hipkiss AR (2005) Glucation, ageing and carnosine: are carnivores' diets beneficial? Mech Ageing Dev 126, 1034-1039.

Hoppe C, Mølgaard C \& Michaelsen KF (2000) Bone size and bone mass in 10-year-old Danish children: effect of current diet. Osteoporos Int 11, 1024-1030.

Hoppe C, Mølgaard C, Juul A \& Michaelsen KF (2004) High intakes of skimmed milk, but not meat, increase serum IGF-I and IGFBP-3 in eight-year-old boys. Eur J Clin Nutr 58, 1211-1216.
Hoppe C, Mølgaard C, Vaag A, Barkholt V \& Michaelsen KF (2005) High intakes of milk, but not meat, increase s-insulin and insulin resistance in 8-year-old boys. Eur J Clin Nutr 59, 393-398.

Lehnert W \& Hunkler D (1986) Possibilities of selective screening for inborn errors of metabolism using high-resolution ${ }^{1} \mathrm{H}$-FT-NMR spectroscopy. Eur Pediatr 145, 260-266.

Lemon PW (2002) Dietary creatine supplementation and exercise performance: why inconsistent results? Can J Appl Physiol 27, $663-680$

Lenz EM, Bright J, Wilson IS, Huges A, Morrison J, Lindberg H \& Lockton A (2004) Metabonomics, dietary influences and cultural differences: a ${ }^{1} \mathrm{H}$ NMR-based study of urine samples obtained from healthy British and Swedish subjects. J Pharm Biomed Anal 36, 841-849.

Lindon J, Holmes E \& Nicholson JK (2006) Metabonomics techniques and applications to pharmaceutical research \& development. Pharm Res 23, 1075-1088.

Lindon JC, Nicholson JK \& Everett JR (1999) NMR spectroscopy of biofluids. Ann Rep NMR Spectr 38, 1-88.

Lukaszuk JM, Robertson RJ, Arch JE, Morre GE, Yaw KM, Kelley DE, Rubin JT \& Moyna NM (2002) Effect of creatine supplementation and a lacto-ovo-vegetarian diet on muscle creatine concentration. Int J Sport Nutr Exerc Metab 12, 336-348.

Martens H \& Dardenne P (1998) Validation and verification of regression in small data sets. Chemometrics Intell Lab Syst $\mathbf{4 4}$, 99-121.

Martens H \& Martens M (2000) Modified jack-knife estimation of parameter uncertainty in bilinear modelling by partial least squares regression (PLSR). Food Qual Preference 11, 5-16.

Mulder TP, Rietvald AG \& van Amelsvoort JM (2005) Consumption of both black tea and green tea results in an increase in the excretion of hippuric acid into urine. Am J Clin Nutr 81, $256 \mathrm{~s}-260 \mathrm{~s}$.

Phipps AB, Stewart J, Wright B \& Wilson ID (1998) Effect of diet on the urinary excretion of hippuric acid and other dietary-derived aromatics in rat. A complex interaction between diet, gut microflora and substrate specificity. Xenobiotica 28, 527-537.

Rolland-Cachera MF, Deheeger M \& Bellisle F (1999) Increasing prevalence of obesity among 18-year-old males in Sweden: evidence for early determinants. Acta Paediatr 88, 365-367.

Solanky KS, Bailey NJC, Holmes E, Beckwith-Hall BM, Davis A, Bingham S, Holmes E, Nicholson JK \& Cassidy A (2003b) Application of biofluid ${ }^{1} \mathrm{H}$ nuclear magnetic resonance-based metabonomic techniques for the analysis of the biochemical effects of dietary isoflavones on human plasma profile. Anal Biochem 323, 197-204.

Solanky KS, Bailey NJC, Holmes E, Lindon JC, Davis AL, Mulder TPJ, Van Duynhoven JPM \& Nicholson JK (2003a) NMR-based metabonomic studies on the biochemical effects of epicatechin in the rat. J Agric Food Chem 51, 4139-4145.

Teegarden D, Lyle RM, McCabe GP, et al. (1998) Dietary calcium, protein, and phosphorus are related to bone mineral density and content in young women. Am J Clin Nutr 68, 749-754.

Wang YL, Tang HR, Nicholson JK, Hylands PJ, Sampson J \& Holmes E (2005) A metabonomic strategy for the detection of the metabolic effects of chamomile (Matricaria recutita L.) ingestion. J Agric Food Chem 53, 191-196.

Williams RE, Eyton-Jones HW, Farnworth MJ, Gallagher R \& Provan WM (2002) Effect of intestinal microflora on the urinary metabolic profile of rats: a ${ }^{1} \mathrm{H}$-nuclear magnetic resonance spectroscopy study. Xenobiotica 32, 783-794.

Wu PSC \& Otting G (2005) SWET for secure water suppression on probes with high quality factor. $J$ Biomol NMR 32, 243-250. 